\title{
Stability Boundaries for Offshore Wind Park Distributed Voltage Control
}

Gryning, Mikkel P.S. ; Wu, Qiuwei; Kocewiak, Lukasz; Niemann, Hans Henrik; Andersen, Karsten P.H.; Blanke, Mogens

Published in:

IEEE Transactions on Control Systems Technology

Link to article, DOI:

10.1109/TCST.2016.2606889

Publication date:

2017

Document Version

Peer reviewed version

Link back to DTU Orbit

Citation (APA):

Gryning, M. P. S., Wu, Q., Kocewiak, L., Niemann, H. H., Andersen, K. P. H., \& Blanke, M. (2017). Stability Boundaries for Offshore Wind Park Distributed Voltage Control. IEEE Transactions on Control Systems Technology, 25(4), 1496 - 1504. https://doi.org/10.1109/TCST.2016.2606889

\section{General rights}

Copyright and moral rights for the publications made accessible in the public portal are retained by the authors and/or other copyright owners and it is a condition of accessing publications that users recognise and abide by the legal requirements associated with these rights.

- Users may download and print one copy of any publication from the public portal for the purpose of private study or research.

- You may not further distribute the material or use it for any profit-making activity or commercial gain

- You may freely distribute the URL identifying the publication in the public portal 


\title{
Stability Boundaries for Offshore Wind Park Distributed Voltage Control
}

\author{
Mikkel P. S. Gryning, Student Member, IEEE, Qiuwei Wu, Senior Member, IEEE, \\ Łukasz Kocewiak, Senior Member, IEEE, Hans Henrik Niemann, \\ Karsten P. H. Andersen, and Mogens Blanke, Senior Member, IEEE
}

\begin{abstract}
In order to identify mechanisms causing slow reactive power oscillations observed in an existing offshore wind power plant (WPP), and be able to avoid similar events in the future, voltage control is studied in this brief for a plant with a static synchronous compensator, type- 4 wind turbines, and a park pilot control. Using data from the actual WPP, all stabilizing subsystem voltage proportional-integral controller parameters are first characterized based on their Hurwitz signature. Inner loop current control is then designed using internal mode control principles, and guidelines for feedforward filter design are given to obtain required disturbance rejection properties. This brief contributes by providing analytical relations between power plant control, droop, sampling time, electrical parameters, and voltage control characteristics, and by assessing frequencies and damping of reactive power modes over a realistic envelope of electrical impedances and control parameters.
\end{abstract}

Index Terms-Reactive power control, voltage control, wind energy integration, wind power plants (WPPs).

\section{INTRODUCTION}

$\mathbf{F}$ ULL-SCALE back-to-back converters are used in large wind turbines (WTs) to control active and reactive power [1]. Power electronic devices enable fast and independent control at the cost of increased complexity of wind power plants (WPPs) and the associated control systems [2]. Offshore turbines are connected to the transmission system through a medium-voltage subsea cable network and a high-voltage network from the point of common coupling (PCC). The WT control system design is intricate due to limited knowledge of the transmission system characteristics, and, depending on the system topology, uncertain interconnection dynamics between distributed voltage controllers can pose a threat to overall

Manuscript received July 8, 2016; accepted August 15, 2016. Manuscript received in final form August 29, 2016. This work was supported in part by DONG Energy and in part by the Danish Council for Technology and Innovation for Funding the Research Project. Recommended by Associate Editor P. Korba.

M. P. S. Gryning is with DONG Energy, 2820 Gentofte, Denmark, and also with the Department of Electrical Engineering, Technical University of Denmark, 2800 Kongens Lyngby, Denmark (e-mail: migry@dongenergy.dk).

Q. Wu and H. H. Niemann are with the Department of Electrical Engineering, Technical University of Denmark, 2800 Kongens Lyngby, Denmark (e-mail: qw@elektro.dtu.dk; hhn@elektro.dtu.dk).

Ł. Kocewiak and K. P. H. Andersen are with DONG Energy, 2820 Gentofte, Denmark (e-mail: lukko@dongenergy.dk; kahan@dongenergy.dk).

M. Blanke is with the Department of Electrical Engineering, Technical University of Denmark, 2800 Kongens Lyngby, Denmark, and also with the Department of Engineering Cybernetics, Norwegian University of Science and Technology, 7491 Trondheim, Norway (e-mail: mb@elektro.dtu.dk).

Color versions of one or more of the figures in this paper are available online at http://ieeexplore.ieee.org.

Digital Object Identifier 10.1109/TCST.2016.2606889

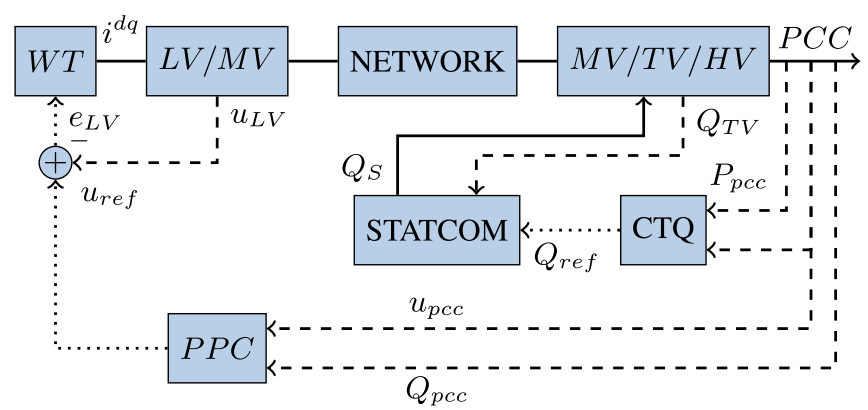

Fig. 1. Schematic wind farm voltage control. Plants $W T$ and STATCOM include current controllers in the blocks. CTQ, control, calculates power set point. Dashed arrows: measurement. Dotted arrows: command. Solid arrows: physical connection.

system stability. This brief was motivated by a stability issue that was encountered when a large WPP was commissioned.

Fig. 1 shows the voltage control topology for the WPP studied here. The PCC voltage control system consists of voltage control at the WT low-voltage (LV) terminals and at the static synchronous compensator (STATCOM). The power plant control (PPC) provides a voltage reference to the WTs, which change reactive power, while the STATCOM operates autonomously. In this case, two systems seek to control the PCC voltage by injecting reactive power at electrically distant points. This could lead to unwanted interactions if the system parameters are not aligned [3].

The design of a voltage control strategy is limited by partial unknown network parameters and system dynamics [4]. If the estimation of the system parameters is poor, local voltage profile regulation can lead to reactive power oscillations [5]. Furthermore, local voltage control may influence voltage flicker, caused by unwanted interaction between individual control loops [6], [7]. An assessment of the static voltage stability of a power system considering all power controls was presented in [8], using singular values, but dynamics of interacting converter systems in a network affect the parameter range of stabilizing controls and instability could occur [9]. An analysis of local regulation of voltage profiles in distributed systems, considering WT capability curves, was treated in [4], who also addressed stability. Static provision of reactive power by a decentralized generation system was studied in [10], and as reactive power capability is a function of active power output [11], combined power optimization and reactive power control were studied in [12]. 
As local voltage is controlled by regulating the balance of reactive power, WPP dynamic voltage stability requires fast distributed reactive sources [13]. Back-to-back or matrix converters are the prevalent technologies used for this control [14], where the output waveform of the converter is a function of grid voltage, and feedforward is used to improve disturbance rejection [15]. The feedforward is low-pass filtered in a tradeoff between noise, over current protection, and stability [16]. Hence, the literature has mainly studied single control loops but has not addressed the entire topology of a WPP system.

Addressing the entire hierarchy of controls that establish the voltage control of a WPP, this brief investigates possible causes of reactive power oscillations based on a case where poorly damped responses were observed. Different mechanisms are identified and stability boundaries are calculated in order to find root causes for the observed behaviors. The probable causes are discussed and a procedure is suggested to obtain robust design of WPP distributed voltage control. It is shown how the complex Hurwitz test, which was used for the analysis of a current controlled generator in [17], can be extended to find the stabilizing parameter space for a complex WPP control with several interacting control loops.

The contributions of this brief are: 1) to describe a systematic approach to finding root causes to an observed reactive power oscillation; 2) to propose a methodology to identify the main contributing factors to the phenomena; 3) identify the stability boundaries of WT voltage source inverter (VSI) and STATCOM control; and 4) to suggest a systematic approach to control design that assures sufficient robustness, such that reactive power oscillations are avoided in new WPPs.

This brief first introduces the gathered data and proposes two possible causes. The methodology used to investigate the propositions is presented in Section III. Section IV focuses on tuning of the WT VSI control. The results from Section IV are applied in Section V to the STATCOM control. Section VI describes the effect of parameter variation on reactive power oscillations. Finally, Section VII discuss the contributory causes of the observed oscillation followed by conclusions.

\section{Problem Formulation}

An incident of unacceptable reactive power oscillations in a WPP was observed. The phenomenon appeared as underdamped oscillations at the PCC shown in Fig. 2. Iterative adjustment of converter controller gains and disconnecting WTs from the PPC attenuated the oscillation amplitude and partly remedied the problem; however, the root cause of the problem was never clarified. The attenuation indicates a problem with the initial tuning of voltage controllers, or an unwanted coupling between the WTs and the STATCOM. This brief seeks to clarify the possible causes of the marginal voltage stability, and provide firm guidelines to be applied during early project analysis and specification for future WPPs. The following propositions reflect possible causes as seen by the WT supplier.

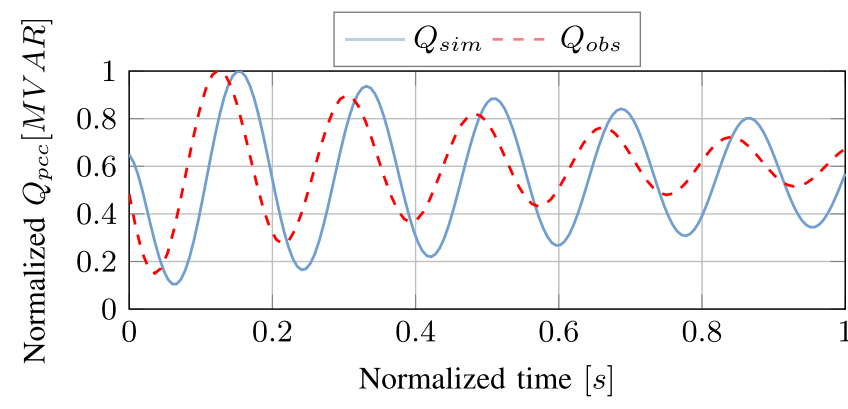

Fig. 2. Simulated reactive power oscillations superimposed on the observed phenomena.

1) Proposition I: Inadequate controller bandwidth separation in the WT voltage control configuration and interaction with grid dynamics.

2) Proposition II: Regulating the PCC voltage by reactive power injection at two electrically distant points in the WPP, using identical feedback signal.

The goal of this brief is to estimate, if any, the level of contribution each proposition has on the observed phenomena. This is achieved through a study of the WPP voltage control structure shown in Fig. 1.

\section{Methodology}

The phenomena can be studied as underdamped oscillations in a stable dynamic system. An understanding of the phenomena and cause hereof is thus obtainable by modeling the implicated systems, and identifying the range of controller parameters for which the system remains stable. As the electrical network and other control loops affect the range of stabilizing controller parameters, an analytical solution is required to establish bounds. From the Hurwitz signature of the closed-loop system, an analytical expression for stability exists based on the number of distinct nonnegative roots of the associated polynomial [18]. The number of distinct nonnegative roots as a function of system parameters is guaranteed by an analytical solution of the Sturm sequence components. The solution is verified numerically by root-locus analysis or time domain simulation in the identified range of controller parameters.

\section{A. Parameter Bounds}

Given a rational polynomial with real coefficients $Q(j \omega)=N(j \omega) / D(j \omega)$ with $N(j \omega)$ having no zeroes on the $j \omega$-axis, the polynomial can be rewritten as

$$
Q(j \omega)=Q_{r}(\omega)+j Q_{i}(\omega)=Q_{\mathcal{E}}\left(-\omega^{2}\right)+\omega Q_{\mathcal{O}}\left(-\omega^{2}\right) .
$$

The total phase change, i.e., the Hurwitz signature, of $Q(j \omega)$ is [19]

$\sigma(Q)=\left(\sum_{j=1}^{l-1}(-1)^{l-1-j} \operatorname{sgn}\left[Q_{r}\left(\omega_{j}\right)\right]\right) \operatorname{sgn}\left[Q_{i}\left(\infty^{-}\right)\right]$ 


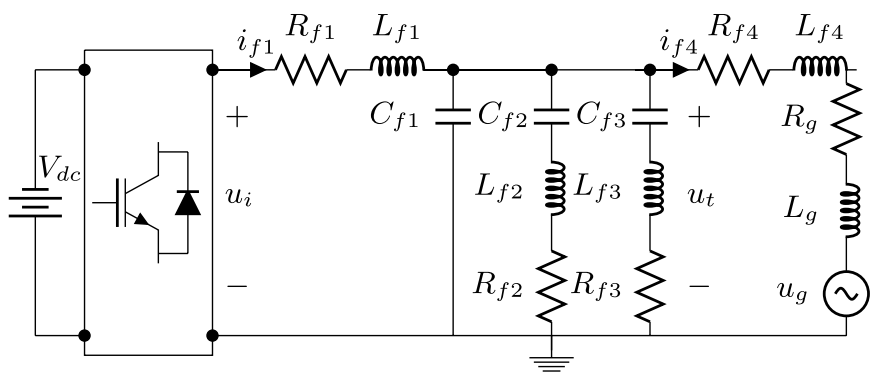

Fig. 3. Single line diagram of VSI with LCL and resistive losses $\left(L_{f 1}\right.$, $C_{f 1}, L_{f 4}$ and $\left.R_{f 1}, R_{f 4}\right)$, trap filter for the first carrier group $\left(C_{f 2}, L_{f 2}\right.$, and $\left.R_{f 2}\right)$ and the second carrier group $\left(C_{f 3}, L_{f 3}\right.$, and $\left.R_{f 3}\right)$ connected to grid.

where $l-1$ is the number of real nonnegative zeroes of $Q_{i}(\omega)$. Let $\operatorname{deg}[D(s)]=n$, $\operatorname{deg}[N(s)]=m \leq n$, and $K(s)=k_{p}+k_{i} / s$; closed-loop stability of the feedback interconnection $Q(s)$ and $K(s)$ is equivalent to having $n+1$ zeroes of the characteristic polynomial $\delta(s)$ in the open left half plane (LHP). It follows:

$$
\sigma(\delta(s)) \geq n+1 .
$$

The polynomial $v(s)=\delta(s) N(-s)$ exhibits the parameter separation property, such that [19]

$$
v(s)=v_{\mathcal{E}}\left(s^{2}, k_{i}\right)+s v_{\mathcal{O}}\left(s^{2}, k_{p}\right) .
$$

Stability of $\delta(s)$ follows from:

$$
\sigma(v(s))=\sigma(\delta(s))+\sigma(N(-s))=(n-m)+1+2 z^{+}
$$

where $z^{+}$denotes the number of right half plane zeroes of $Q(s)$. The Hurwitz signature is expanded as

$$
\begin{aligned}
\sigma(v) & =j\left(i_{0}-2 i_{1}+\cdots+(-1)^{l} 2 i_{l}+(-1)^{l+1} i_{l+1}\right) \\
j & =\operatorname{sign}\left(v_{\mathcal{O}}\left(0^{+}, k_{p}^{*}\right)\right)
\end{aligned}
$$

and let $\boldsymbol{\Gamma}$ define the distinct strings of integers $\left\{i_{0}, i_{1}, \ldots, i_{l+1}\right\}$ satisfying (5). The stabilizing set of integral gains $k_{i}$, given a $k_{p}$, such that $v_{\mathcal{O}}$ has $l$ distinct nonnegative zeroes, is given by the linear inequalities [19]

$$
v_{\mathcal{E}}\left(-\omega_{t}^{2}, k_{i}\right) i_{t}>0
$$

where $i_{t}$ ranges over each of the strings in $\Gamma$ and $\omega_{t}$ is the associated root of $v_{\mathcal{O}}$. The minimum number of distinct nonnegative roots to satisfy (5) is

$$
l \geq(n-m+1) / 2-1 .
$$

The full stabilizing set $\boldsymbol{\Omega}\left(k_{p}, k_{i}\right)$ can thus be found by guaranteeing $l$ distinct nonnegative roots of $v_{\mathcal{O}}$ and solve the set of linear inequalities. It is proposed in this brief to extend the stabilizing sets theory by guaranteeing the required number of distinct nonnegative real roots by manipulation of the Sturm sequence. The number of distinct nonnegative real roots in an univariate polynomial in the interval $[0, \infty]$ can be determined by the number of sign changes in the Sturm sequence evaluated at the end points of the interval [20]. Let $p_{1}$ be the number of sign changes at $\omega_{0} \triangleq 0$ and $p_{2}$ at $\omega_{l+1} \triangleq \infty$; the number of distinct nonnegative real roots is $l=p_{1}-p_{2}$ [20].

\section{Proposition I: Wind Turbine Voltage Control}

A model of the WT voltage control and the effect of parameter variation on system modes are investigated in this section. Bounds on the current control parameters are established for proper performance and disturbance rejection, and subsequently used in the voltage control analysis.

\section{A. System Model}

A VSI with an inductor-capacitor-inductor (LCL) and trap filters is shown in Fig. 3. The LCL-filter is approximated by an inductor filter for low frequencies [21]. The filters and the transformer represent the plant $G_{1}(s)$, and the grid is the disturbance dynamics $G_{d}(s)$. The current loop dynamics are given by

$$
L_{f} \frac{d i_{f}^{d q}}{d t}+R_{f} i_{f}^{d q}=u_{i}^{d q}-E^{d q}+D L_{f} D \omega_{g} i_{f}^{d q}
$$

where

$$
\begin{aligned}
\boldsymbol{\omega}_{\boldsymbol{g}} & =\left[\begin{array}{cc}
0 & \omega_{g} \\
-\omega_{g} & 0
\end{array}\right], \quad \boldsymbol{D}=\left[\begin{array}{ll}
0 & 1 \\
1 & 0
\end{array}\right] \\
\boldsymbol{L}_{\boldsymbol{f}} & =\boldsymbol{L}_{\boldsymbol{f} \mathbf{1}}+\boldsymbol{L}_{\boldsymbol{f} \mathbf{4}}+\boldsymbol{L}_{\boldsymbol{g}}, \quad \boldsymbol{R}_{\boldsymbol{f}}=\boldsymbol{R}_{\boldsymbol{f} \mathbf{1}}+\boldsymbol{R}_{\boldsymbol{f} \mathbf{4}}+\boldsymbol{R}_{\boldsymbol{g}} .
\end{aligned}
$$

The rotating reference frame (RRF) axis coupling and the grid voltage disturbance $\boldsymbol{E}^{d \boldsymbol{q}}$ are canceled by introducing feedback of the output current $i_{f}^{d q}$ and feedforward of the measured grid disturbance $\boldsymbol{E}_{\boldsymbol{m}}^{\boldsymbol{d q}}$ as

$$
u_{i *}^{d q}=u_{i}^{d q}-C(s) E_{m}^{d q}+D L_{f} D \omega_{g} i_{f m}^{d q}
$$

where the measured grid voltage is filtered by the low-pass filter $C(s)=\alpha_{f} /\left(s+\alpha_{f}\right), \omega_{g}$ is the angular frequency of the grid, and $\alpha_{f}$ is the filter tuning variable. The low frequency resonance characteristic is damped using the virtual resistor principle with resistance $R_{d}$ [22]. Given a bounded reference trajectory $u_{i}^{r}(t)$, the computational and switching delay is modeled as a dead time $\tau_{s}$

$$
u_{i}(t)=e^{-s \tau_{s}} u_{i}^{r}(t)
$$

and the system is cast as two identical single input-single output systems. In the Laplace domain

$$
u_{i}(s)=\frac{u_{i}^{r}(s)}{\tau_{s} s+1}, i_{f}(s)=\frac{u_{i}(s)-E(s)}{L_{f} s+R_{f}} .
$$

The voltage at the LV terminals of the WT transformer $u_{t}(s)$ as a function of output current $i_{f}(s)$ and grid voltage $E(s)$ with scaling variable $\lambda$ to account for losses in the filter is

$$
u_{t}(s)=\frac{\left(L_{v} s+R_{v}\right) i_{f}(s)+E(s)}{C_{p} L_{v} s^{2}+C_{p}\left(R_{v}+\left(R_{d} / \lambda\right)\right) s+1}
$$

where $L_{v}=L_{f}-L_{f 1}$ and $C_{p}=C_{f 1}+C_{f 2}+C_{f 3}$. 


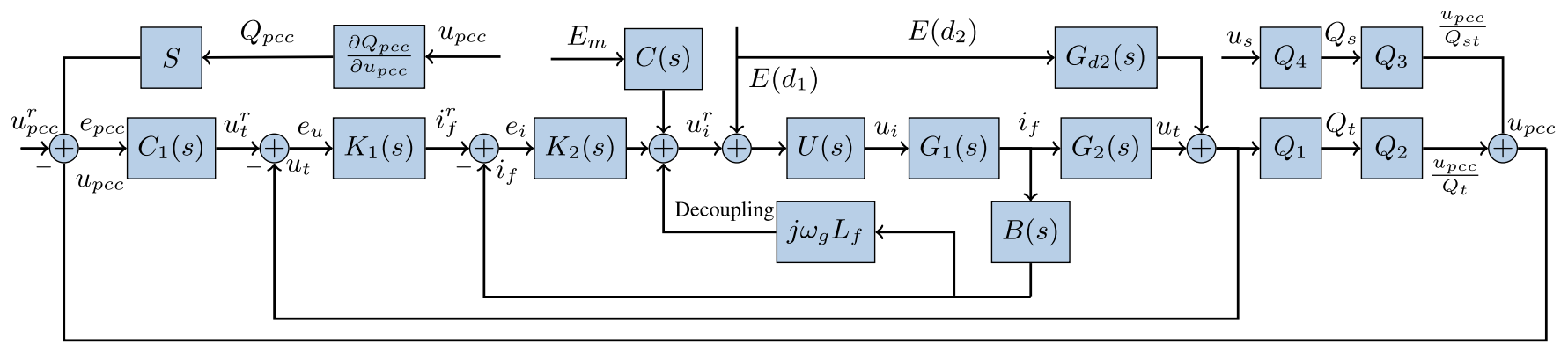

Fig. 4. Cascade voltage control structure of the VSI. The PI controller $K_{2}(s)$, actuator $U(s)$, and plant $G_{1}(s)=i_{f 4} / u_{i}$ constitute the inner current control loop with output disturbance $G_{d}(s)=i_{f 4} / u_{g}$. The outer loop consists of a PI controller $K_{1}(s)$, the inner loop, and $G_{2}(s)=u_{t} / i_{f 4}$. The voltage sensitivities are $Q_{1}=\partial Q_{t} / \partial u_{t}, Q_{2}=\partial u_{p c c} / \partial Q_{t}, Q_{3}=\partial u_{p c c} / \partial Q_{s}$, and $Q_{4}=\partial Q_{s} / \partial u_{s}$, with $u_{s}$ being the STATCOM terminal voltage. $E_{m}$ is the measured grid voltage, and $E(d 1)$ and $E(d 2)$ are the grid disturbances on the current control and inverter voltage control, respectively.

\section{B. Current Control Design}

The current control structure consists of a proportionalintegral (PI) controller for zero steady state error and a notch filter, $B$, as shown in Fig. 4. Let $e_{i}=i_{f}^{r}-i_{f}$ and $K_{2}(s)=k_{p c}+\frac{k_{i c}}{s}$; the reference voltage is

$$
u_{i}^{r}=K_{2}(s)\left(e_{i}\right)+C(s) E \pm j \omega_{g} L_{f} i_{f} .
$$

The closed-loop transfer functions from reference $i_{f}^{r}(s)$ to output $i_{f}(s)$ and from disturbance $E(s)$ to $i_{f}(s)$ are given by

$$
\begin{aligned}
i_{f}(s) & =\frac{\left(k_{p c} s+k_{i c}\right) i_{f}^{r}(s)}{D(s)}-\frac{\left(s \tau_{s}+1\right) s^{2} E(s)}{\left(s+\alpha_{f}\right) D(s)} \\
D(s) & =L_{f} \tau_{s} s^{3}+\left(R_{f} \tau_{s}+L_{f}\right) s^{2}+\left(B k_{p c}+R_{f}\right) s+B k_{i c} .
\end{aligned}
$$

The system for control synthesis is represented as a first-order plus time delay model, $\bar{G}_{1}(s)$

$$
\bar{G}_{1}(s)=\frac{1}{R_{f}} \frac{1}{\left(L_{f} / R_{f}+\tau_{s} / 2\right) s+1} e^{-\tau_{s} / 2 s} .
$$

By process inversion design, the controller parameters are given as [23]

$$
\begin{aligned}
k_{p c} & =\left(L_{f}+R_{f}\left(\tau_{s} / 2\right)\right) /\left(\tau_{c}+\tau_{s} / 2\right) \\
k_{i c} & =k_{p c} /\left(\min \left(L_{f} / R_{f}+\tau_{s} / 2,4\left(\tau_{c}+\tau_{s} / 2\right)\right)\right)
\end{aligned}
$$

where $\tau_{c}$ is a tuning parameter for tradeoff between output performance and robustness.

1) Parameter Bounds: The system bandwidth is constrained by the switching frequency $f_{s}$, attenuation of harmonics, maximum gain $K_{\max }$, and system resonances [24]. Assuming small $k_{i c}$, (20), and the closed-loop bandwidth tradeoff, $f_{b}=0.2 f_{s}$, lower bounds $\tau_{c}$ for performance [25]

$\tau_{c} \geq \begin{cases}\frac{-50\left(\kappa_{2}+L_{f}\right) R_{f}-\tau_{s} \kappa_{1}+5 \kappa_{2} \kappa_{3}+10 L_{f} \kappa_{3}}{50 R_{f}^{2}+2 \kappa_{1}}, & f_{s} / 5 \\ \frac{\left(\tau_{s} / 2\right)\left(R_{f}-K_{\max }\right)+L_{f}}{K_{\max }}, & k_{p c} \leq K_{\max }\end{cases}$

where $\kappa_{1}-\kappa_{9}$ are parameters given in [26]. Selecting $\tau_{c}$ from bandwidth limitation of $k_{p c}$ gives an upper bound on $k_{i c}$

$$
k_{i} \leq \frac{\left(25 R_{f}^{2}+\kappa_{1}\right)^{2}}{50\left(\kappa_{3}-5 R_{f}\right)^{2}\left(\kappa_{2}+2 L_{f}\right)} .
$$

2) Disturbance Rejection: The choice of low-pass filter coefficient $\alpha_{f}$ determines the bandwidth. Converter over current protection requires small $\left|i_{f}^{\max }\right|$ for network disturbances [27]. This is achieved with large $\alpha_{f}$. Parameters $\alpha_{f}$ and $k_{p}$ are, respectively, lower and upper bounded by bandwidth limitations. For sufficiently small $\tau_{s}<1 \mathrm{~ms}$, the roots of $D(s)$, $z_{p}$, are governed by

$$
z_{p} \in \mathbb{R}, \quad k_{i c}<\left(R_{f}^{2}+k_{p c}^{2}+2 R_{f} k_{p c}\right)\left(4 L_{f}\right)^{-1} .
$$

Bounding $k_{i c}$ by (24) guarantees a damping of unity on all modes. The system response to a grid voltage step disturbance as a function of PI parameters and $\alpha_{f}$ is

$$
i(t)=\frac{\left(\left(\kappa_{9}-\kappa_{8}\right) \mathrm{e}^{2 \kappa_{7} t}+\kappa_{9}+\kappa_{8}\right) \mathrm{e}^{-t\left(\kappa_{4}+\kappa_{7}\right)}-2 \mathrm{e}^{-t \alpha_{f}} \kappa_{9}}{-L_{f}^{-1} \kappa_{6}} .
$$

The response has a global maximum at

$$
t_{\max }=\ln \left(\frac{-2 \kappa_{5}}{\kappa_{2}-\kappa_{3}}\right) /\left(\alpha_{f}-\kappa_{4}-\kappa_{7}\right) .
$$

If restrictions on $t_{\max }$ or $\left|i_{f}^{\max }\right|$ exist, $\alpha_{f}$ should be selected from (26) or a contour plot of (25).

\section{Voltage Control Analysis}

The structure of $K_{1}(s)=k_{p v}+k_{i v} / s$ is a PI controller. The reference signal is

$$
i_{f}^{r}(s)=K_{1}(s)\left(u_{t}^{r}(s)-u_{t}(s)\right) .
$$

The closed-loop system defined by (16) and (18) with input (27) has $n=5$ and $m=2$, which satisfies (9) for $l \geq 1$. The Sturm sequence $\Psi(\omega)$ of $v_{\mathcal{O}}\left(\omega_{0}, k_{p v}\right)$ consists of four subsequent equal constants $\alpha_{0}$ with alternating signs

$$
\alpha_{0}=k_{p v}+1 / R_{v}
$$

such that for $k_{p v} \in \mathbb{R} \backslash\left\{-1 / R_{v}\right\}, p_{1}=3 \forall k_{p v}$. The Sturm sequence of $v_{\mathcal{O}}\left(\infty, k_{p v}\right)$ is

$$
\Psi\left(\infty, k_{p v}\right)=\left\{\alpha_{1}, \alpha_{1}, \alpha_{3}, \alpha_{3}, \alpha_{5}, \alpha_{5}, \alpha_{0}\right\}
$$

where $\alpha_{1}, \alpha_{3}$, and $\alpha_{5}$ are functions of system component parameters and inner loop tuning given in [26]. The lower 


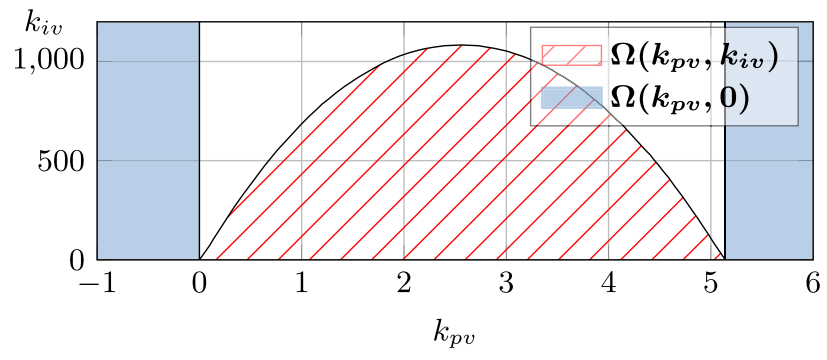

Fig. 5. Stabilizing set $\boldsymbol{\Omega}\left(k_{p v}, k_{i v}\right)$.

bound on $k_{p v}$ follows from manipulation of the $\Psi\left(\infty, k_{p v}\right)$ components, such that $p_{2} \leq 2$ :

$$
k_{p v}>\frac{k_{i c}\left(L_{f} R_{v}-L_{v} R_{f}\right)-k_{p c}\left(R_{v}\left(R_{f}+k_{p c}\right)\right)}{k_{i c}^{2} L_{v}^{2}+k_{p c}^{2} R_{v}^{2}} .
$$

For $l=1$, every string $\boldsymbol{\Gamma}=\left\{i_{0}, i_{1}, i_{2}\right\}$ must satisfy

$$
\operatorname{sign}\left(\nu_{\mathcal{O}}\left(\omega_{0}, k_{p v}\right)\right)\left(i_{0}-2 i_{1}-i_{l+1}\right)=n-m+1
$$

for all $k_{p v}$ in the stabilizing set $\boldsymbol{\Omega}\left(k_{p v}, k_{i v}\right)$. The sign of $\nu_{\mathcal{O}}\left(0^{+}, k_{p v}\right)$ is strictly positive given

$$
\nu_{\mathcal{O}}\left(0^{+}, k_{p v}\right)>0 \forall k_{p v}>-1 / R_{v}
$$

and the magnitude of the bound in $(30) \ll(32)$. Hence, the only admissible string is $\boldsymbol{\Gamma}_{1}=\{1,-1,-1\}$. Following (8), $k_{i v}$ must satisfy the constraints:

$$
\begin{gathered}
p_{1}\left(\omega_{1}\left(k_{p v}\right)\right)+k_{i v} p_{2}\left(\omega_{1}\left(k_{p v}\right)\right)<0 \\
0 \leq k_{i v} \leq \infty .
\end{gathered}
$$

The full stabilizing set $\boldsymbol{\Omega}\left(k_{p v}, k_{i v}\right)$ is found by the variation of $k_{p v}$ in (33). For $k_{p v}$ bounded by (30), the closed-loop system is guaranteed to have one real root. This root, $\omega_{1}\left(k_{p v}\right)$, is found by factorization as

$$
\omega_{1}\left(k_{p v}\right)^{2} \approx \frac{-k_{p c}\left(L_{v} k_{p c} k_{p v}+L_{f}\right)}{L_{v} C_{p}\left(-k_{p c}\left(L_{f}+R_{f} \tau_{s}\right)+L_{f} \tau_{s} k_{i c}\right.} .
$$

The stabilizing set $\boldsymbol{\Omega}\left(k_{p v}, k_{i v}\right)$ is thus the area

$$
k_{i v}<\frac{-p_{1}\left(\omega_{1}\left(k_{p v}\right)\right)}{p_{2}\left(\omega_{1}\left(k_{p v}\right)\right)}, k_{p v} \in \boldsymbol{\Omega}\left(k_{p v}, k_{i v}\right)
$$

for $k_{p v}$ lower bounded by the intersection of (30). Inserting $\omega_{1}\left(k_{p v}\right)$ in (33) upper bounds $k_{p v}$ as the solution to the polynomial

$$
\gamma_{1} k_{p v}^{2}+\gamma_{2} k_{p v}+\gamma_{3}=0
$$

where $\gamma_{1}-\gamma_{3}$ are the functions of system parameters given in [26]. The full stabilizing set $\boldsymbol{\Omega}\left(k_{p v}, k_{i v}\right)$ is shown in Fig. 5.

\section{Conclusion: Proposition I}

Variation of $k_{p v}$ in $\boldsymbol{\Omega}\left(k_{p v}, k_{i v}\right)$ is shown in the root-locus diagram of Fig. 6 . At the boundary of $\boldsymbol{\Omega}\left(k_{p v}, k_{i v}\right)$, the system becomes marginally stable, validating the set. The current control loop contributes with poles $p_{3,6}$, which stay in the LHP for all $k_{p v}$ and $k_{i v}$. Increasing $k_{p c}$ in violation with (24) produces a complex pole pair that increase $\mathbb{R}\left(p_{4,5}\right)$, enlarging $\boldsymbol{\Omega}\left(k_{p v}, k_{i v}\right)$. The voltage control locus of $p_{4,5}$ shows that a

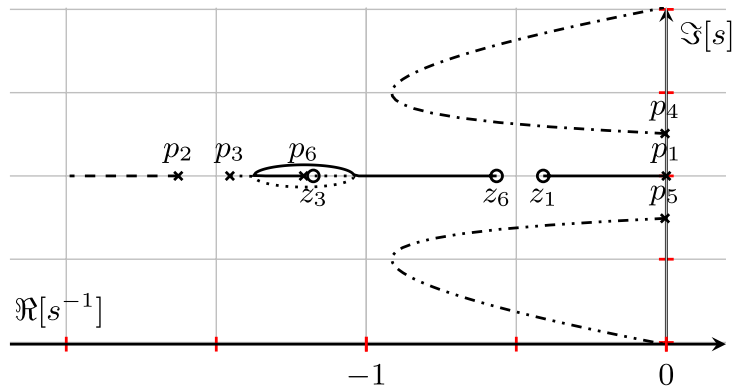

Fig. 6. Scaled root-locus diagram of the open-loop plant for $k_{p v} \in \boldsymbol{\Omega}\left(k_{p v}, k_{i v}\right)$. The left-most pole is stopped at $z_{2}$ for clarity in the central region.

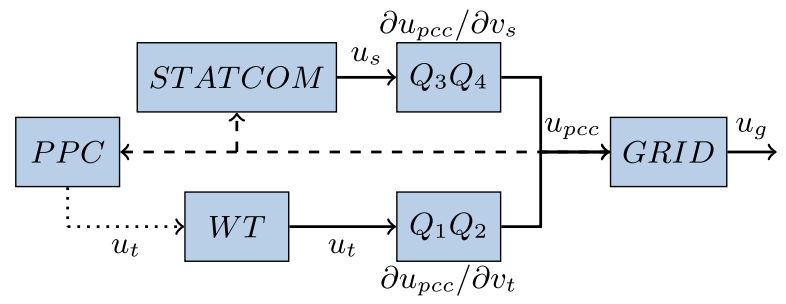

Fig. 7. Conceptional park level voltage control. STATCOM and WT represent structures as Fig. 4. Dashed arrows: measurement. Dotted arrow: command. Solid arrows: physical connection.

desired damping can be obtained by two values of $k_{p v}$, and that increasing $k_{i v}$ shifts the zero $z_{1}$ into the LHP rendering $\mathbb{R}\left(p_{4,5}\right)$ smaller.

If the current control parameters were modified, resulting in a smaller $\boldsymbol{\Omega}\left(k_{p v}, k_{i v}\right)$, unaltered voltage control parameters could provoke under damped oscillations. Furthermore, the combination of a small $k_{i v}$ and a large $k_{p v}$ could contribute to the observed phenomena.

\section{PROPOSITION II}

The PCC voltage is regulated by reactive power from the WTs and STATCOM. The PPC provides the voltage reference to the WT control, as shown in Fig. 7. The bandwidth of the PPC is low compared with the electrical subsystem, which thus is considered a first-order system. In this section, analytical bounds are found for the PPC and the STATCOM using the current control design of Section IV. Combining bounds from Sections IV and V constitute the controller parameters for which the system is stable, and the effect of system parameter change, e.g., cable length, is then simulated for all values in the set to estimate the contribution from Proposition II.

\section{A. System Model}

The WPP characteristics are modeled as power flows described by two $\Pi$ realizations [28]. The equations are linearized, and the weak coupling between reactive power $Q$ and load angle $\theta$ is neglected [29]. Let subscripts $s$ and $t$ denote STATCOM and WT; the voltage sensitivities $Q_{1}-Q_{4}$ 
are

$$
\begin{aligned}
\frac{\partial Q_{t}}{\partial u_{p c c}} & =u_{t} f_{q}\left(Z_{t 1}, Z_{t 2}, 1 / Y_{t}\right)=Q_{2}^{-1}, \quad u_{t} \approx 1 p u \\
\frac{\partial Q_{s}}{\partial u_{p c c}} & =u_{s} f_{q}\left(Z_{s 1}, Z_{s 2}, 1 / Y_{s}\right)=Q_{3}^{-1}, \quad u_{s} \approx 1 p u \\
\frac{\partial Q_{t}}{\partial u_{t}} & =Q_{1}, \quad \frac{\partial Q_{s}}{\partial u_{s}}=Q_{4}
\end{aligned}
$$

where $Q_{t}$ and $Q_{s}$ are the reactive power flows. The sending end, subscript 1 , and receiving end, subscript 2 , impedances and admittances are $Z_{s 1, t 1}, Z_{s 2, t 2}, Y_{s}$, and $Y_{t}$, and $f_{q}(\cdot)$ is a frequency-dependent function given in [26].

\section{B. Power Plant Control}

The PPC is a digital droop plus PI controller with sample time $T_{s}>100 \mathrm{~ms}$ and the control law

$$
u_{p c c}^{d_{e}}=u_{p c c}^{d_{*}}-u_{p c c}^{d}-\left(3 S Q_{p c c}\right) /\left(2 n_{w t} Q_{\max }\right)
$$

where $S$ is the desired slope of the droop control, $n_{w t}$ is the amount of turbines connected, and $Q_{\max }$ is the reactive power capability of one turbine. The reactive power error at the PCC and the output of the WT are [30]

$$
Q_{p c c}^{e}=(3 / 2)\left(-u_{p c c}^{d_{e}} i_{p c c}^{q}\right), \quad Q_{w t}=(3 / 2)\left(-u_{t}^{d_{i}} i_{w t}^{q}\right) .
$$

PI control parameters are found by equating $Q_{w t}$ to $\left(1 / n_{w t}\right) Q_{p c c}^{e}$ and adding the integration parameter $\gamma$

$$
C_{3}(s)=k_{p u}+\frac{k_{i u}}{s}=\frac{i_{p c c}^{q}}{n i_{w t}^{q}} u_{p c c}^{d_{e}}+\frac{\gamma}{s}
$$

where the currents are the rated values. Let $T_{v}$ be the effective rise time of the inner processes and let the discrete zero order hold plus sampler system in the continuous domain be

$$
T(s)=\left(1-e^{-s T_{s}}\right) /\left(T_{s} s\right)
$$

then setting $Q_{c}=Q_{1} Q_{2}$, using a first-order Padé time delay approximation and introducing $\alpha=T_{S} / T_{v}$ as the response time ratio, the open-loop response is

$$
u_{p c c}(s)=Q_{c} /\left(\left(T_{v} s+1\right)\left(\alpha T_{v} / 2 s+1\right)\right) .
$$

The number of distinctive nonnegative real roots needed to satisfy (7) to guarantee stability is $l \geq 1$ for $n=2$ and $m=0$. One nonnegative real root for the Sturm sequence given $v_{\mathcal{O}}\left(\omega_{0}, k_{p u}\right)$ and $v_{\mathcal{O}}\left(\infty, k_{p u}\right)$ is achieved for

$$
k_{p u} \geq-\frac{1}{Q_{c}\left(S Q_{4}+1\right)}, \quad 2 Q_{c}^{2}\left(S Q_{4}+1\right)>0 .
$$

The root is located at

$$
\omega_{1 u}\left(k_{p u}\right)=\sqrt{2} \sqrt{\alpha\left(1+k_{p u}\left(S Q_{4}+1\right) Q_{c}\right)} /\left(\alpha T_{v}\right) .
$$

Every admissible string for $l=1, \Gamma=\left\{i_{0}, i_{1}\right\}$, must satisfy

$$
\operatorname{sign}\left(\nu_{\mathcal{O}}\left(0, k_{p u}\right)\right)\left(i_{0}-2 i_{1}\right)=n-m+1=3
$$

where $\operatorname{sign}\left(v_{\mathcal{O}}\left(0, k_{p u}\right)\right)$ is strictly positive given (46). The only admissible string is $\boldsymbol{\Gamma}_{2}=\{1,-1\}$, imposing the nontrivial constraint for $\omega_{1 u}\left(k_{p u}\right)$

$$
p_{1}\left(\omega_{1 u}\left(k_{p u}\right)\right)+k_{i u} p_{2}\left(\omega_{1 u}\left(k_{p u}\right)\right)<0
$$

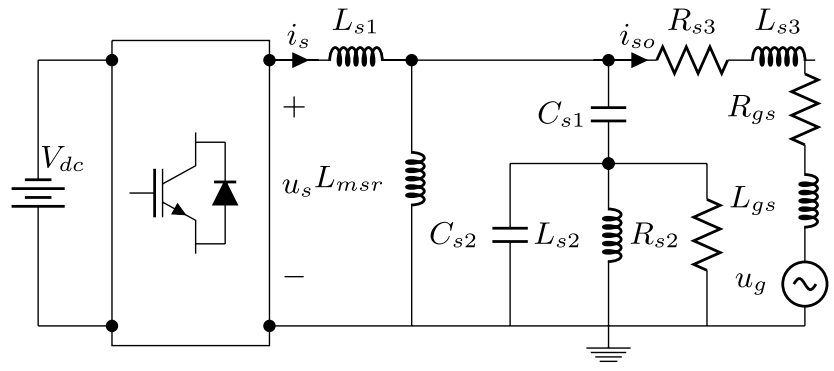

Fig. 8. SLD of grid-connected STATCOM with reactor $\left(L_{\mathrm{msr}}\right)$, harmonic attenuation filters $\left(C_{s 1}, C_{s 2}, L_{s 2}\right.$, and $\left.R_{s 2}\right)$, and onshore transformer represented by $R_{s 3}$ and $L_{s 3}$.

and by insertion of (47) in (49)

$$
k_{i u}<\underbrace{\frac{\alpha+2}{\alpha T_{v}}}_{a_{k}} k_{p u}+\underbrace{\frac{\alpha+2}{\alpha T_{v}} \frac{1}{Q_{c}\left(S Q_{4}+1\right)}}_{b_{k}}
$$

which defines the set $\boldsymbol{\Omega}_{\boldsymbol{H}}\left(k_{p u}, k_{i u}\right)$. Inequality (50) shows $a_{k} \rightarrow 1 / T_{v}$, for $\alpha \rightarrow \infty$, due to increased separation of control and plant bandwidth. An increase of integration gain is needed to guarantee stability as $a_{k} \rightarrow \infty$ for $\alpha \rightarrow 0$. Increasing the sensitivity to reactive power change, $Q_{1}$ to $Q_{4}$, or the droop $S$ decreases the constant factor $b_{k}$.

\section{Dynamic Reactive Power Compensation}

The STATCOM shown in Fig. 8 consists of a reactor and an output filter. A droop plus PI controller regulates the PCC voltage by modifying the STATCOM output current, $i_{s o}(s)$, similar to the VSI with an additional reactor. Decoupling the RRF axes and neglecting high-frequency filter components

$$
i_{s o}(s)=\frac{L_{\mathrm{msr}} u_{i}(s)-L_{d} E(s)}{L_{s} s+R_{s} L_{d}}
$$

where $u_{i}(s)$ is terminal voltage, $E(s)$ is the grid voltage, and

$$
\begin{aligned}
& L_{s}=L_{\mathrm{msr}}\left(L_{s 3}+L_{\mathrm{gs}}+L_{s 1}\right)+\left(L_{s 3}+L_{\mathrm{gs}}\right) L_{s 1} \\
& L_{d}=L_{s 1}+L_{\mathrm{msr}}, \quad R_{s}=R_{\mathrm{gs}}+R_{s 3}
\end{aligned}
$$

are constants. The output filter is modeled with output current $i_{s o}(s)$ and converter voltage $u_{i}(s)$ as inputs and the voltage at the LV side of STATCOM transformer $u_{s t}(s)$ as output

$$
u_{s t}(s)=\frac{\left(n_{1} s+n_{2}\right) L_{\mathrm{msr}}\left(u_{i}(s)-L_{s 1} i_{s o}(s)\right)}{d_{1} s^{4}+d_{2} s^{3}+d_{3} s^{2}+d_{4} s+d_{5}}
$$

where $d_{1}$ to $d_{5}, n_{1}$ and $n_{2}$ are given in [26]. The control law is

$$
\begin{aligned}
i_{s o}^{r}(s) & =K_{3}(s)\left(u_{s t}^{r}(s)-u_{s t}(s)\right) \pm j \omega_{g} C_{p 2} u_{s t}(s) \\
C_{p 2} & =C_{s 1} C_{s 2} /\left(C_{s 1}+C_{s 2}\right)
\end{aligned}
$$

where $K_{3}(s)=k_{\mathrm{psv}}+k_{i s v} / s$. The Sturm sequence of $v_{\mathcal{O}}\left(\omega_{0}, k_{\mathrm{psv}}\right)$ is

$$
\Psi\left(\omega_{0}, k_{\mathrm{psv}}\right)=\left\{l_{0}, 0,-l_{0}, 0, l_{0}, 0,-l_{0}\right\}
$$

where the sequence is alternating with $l_{0}$ as a function of $k_{\mathrm{psv}}$ if

$$
k_{\mathrm{psv}} \in \mathbb{R} \backslash \frac{L_{\mathrm{msr}}\left(n_{2} d_{3}-n_{1} d_{4}\right) k_{i c}+L_{d} R_{s} n_{2} d_{4}}{L_{\mathrm{msr}} k_{i c} n_{2}^{2}} \triangleq l_{\alpha} .
$$




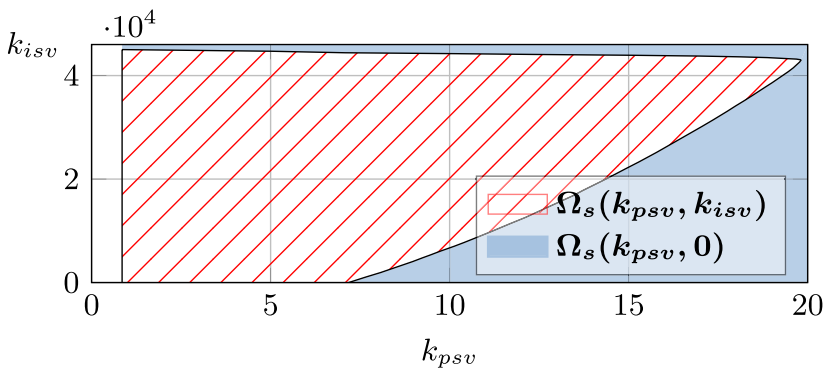

Fig. 9. Stabilizing set $\boldsymbol{\Omega}_{\boldsymbol{S}}\left(k_{\mathrm{psv}}, k_{i s v}\right)$. White area $l_{\mathrm{alpha}}<k_{\mathrm{psv}}<\gamma_{2}$, hatched area $\boldsymbol{\Omega}_{\boldsymbol{s}}\left(k_{\mathrm{psv}}, k_{i s v}\right)$, and blue area $\boldsymbol{\Omega}_{\boldsymbol{s}}\left(k_{\mathrm{psv}}, 0\right)$.

The sequence for $v_{\mathcal{O}}\left(\infty, k_{\mathrm{psv}}\right)$ is given by

$$
\Psi\left(\infty, k_{\mathrm{psv}}\right)=\left\{\alpha_{7}, \alpha_{7}, \alpha_{8}, \alpha_{9}, \alpha_{9}, \alpha_{10}, \alpha_{11}, 0\right\}
$$

where $\alpha_{7}-\alpha_{11}$ and $\gamma_{2}$ are given in [26]. For $n=6$ and $m=3$, $l \geq 1$ to satisfy (7). This is achieved for

$$
l_{\alpha}<k_{\mathrm{psv}}<\gamma_{2}, \quad k_{p c}>0, k_{i c}>0 .
$$

The two admissible strings satisfying (9) are $\Gamma_{3}=$ $\{1,-1,1,1\}$ and $\boldsymbol{\Gamma}_{4}=\{-1,-1,1,-1\}$, imposing constraints

$$
\begin{aligned}
& S_{1}: p_{1}\left(\omega_{2}\left(k_{\mathrm{psv}}\right)\right)+k_{i s v} p_{2}\left(\omega_{2}\left(k_{\mathrm{psv}}\right)\right)>0 \\
& S_{2}: p_{1}\left(\omega_{3}\left(k_{\mathrm{psv}}\right)\right)+k_{i s v} p_{2}\left(\omega_{3}\left(k_{\mathrm{psv}}\right)\right)<0
\end{aligned}
$$

where $\omega_{2}\left(k_{\mathrm{psv}}\right)$ and $\omega_{3}\left(k_{\mathrm{psv}}\right)$ are the isolated roots. The envelope of stability for the STATCOM control, $\boldsymbol{\Omega}_{\boldsymbol{s}}\left(k_{\mathrm{psv}}, k_{i s v}\right)=$ $S_{1} \cup S_{2}$, is shown in Fig. 9.

\section{Vi. Parameter Effect Simulation}

Sections IV-C, V-B, and V-C estimated the control parameters for which each individual system is stable. The effect of control parameters on reactive power oscillations at the PCC is studied by time domain simulation of the entire WPP. Each parameter vector $\boldsymbol{\Psi}_{\boldsymbol{i}}$ of the joint stabilizing set $\boldsymbol{\Omega}_{\boldsymbol{M}}(\cdot)$ is applied, and the system is subjected to a step in grid voltage. The export cable length is varied from $10 \%$ to $300 \%$ of nominal, defining the set $L_{\Omega}$ for all $\boldsymbol{\Psi}_{\boldsymbol{i}}$.

The dominating frequencies are identified from peaks in the estimated spectral density of the response. The damping of the identified modes is found by time shifting and applying the moving block method [31]. The i'th frequency, maximum peak, and damping matrix $\left[\boldsymbol{f}_{i}, \boldsymbol{A}_{\boldsymbol{i}}, \zeta_{i}\right]$ are sorted descending with respect to maximum peak, and each simulation is represented by two scalars

$$
\begin{aligned}
f_{m, i} & =\sum_{j=1}^{n}\left(f_{i, j} A_{i, j}\right) / A_{i, \text { avg }} \\
\zeta_{m, i} & =\sum_{j=1}^{n}\left(\zeta_{i, j} A_{i, j}\right) / A_{i, \text { avg }}
\end{aligned}
$$

where $A_{i, \text { avg }}=\sum_{j=1}^{n}\left(A_{i, j}\right)$. The results are visualized in a 3-D bar plot where the $y$-axis is the variation of cable length in $\boldsymbol{L}_{\mathbf{\Omega}}$, the $x$-axis shows the control parameters to be varied, the $z$-axis is the variation of the parameters within the joint stabilizing set in percent, and the colors represent the damping.

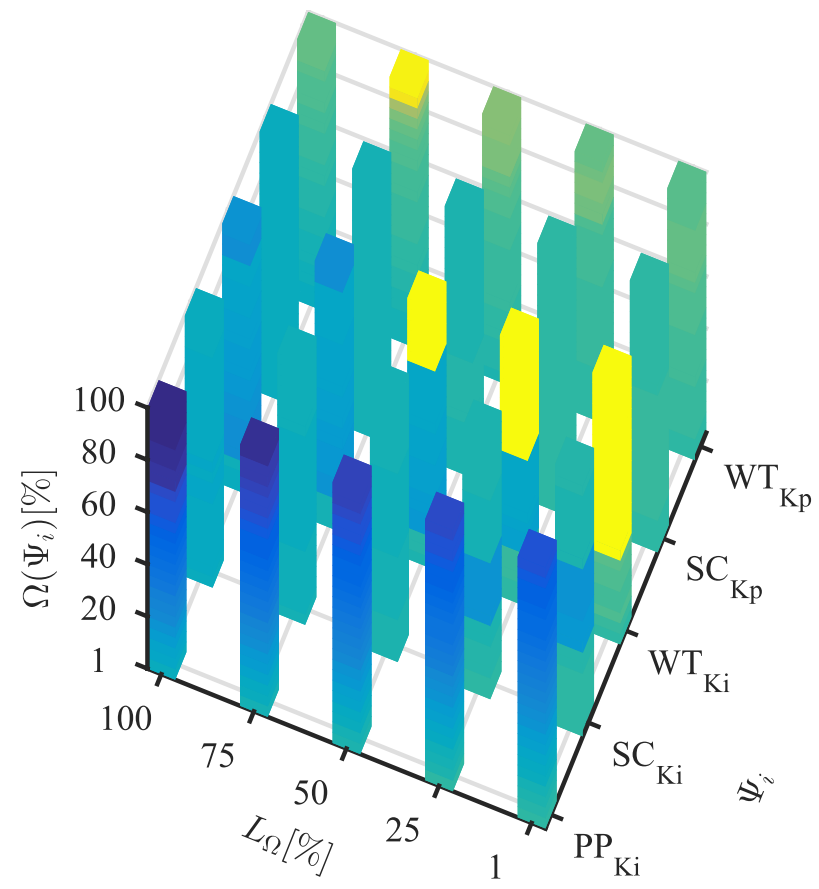

\section{$\begin{array}{lllllllll}0.1 & 0.2 & 0.3 & 0.4 & 0.5 & 0.6 & 0.7 & 0.8 & 0.9\end{array}$ Damping [.]}

Fig. 10. Amplitude weighed damping from variation of cable length in $L_{\Omega}$ versus each controller parameter in the vector $\boldsymbol{\Psi}_{i}$ and their variation in the stable set $\boldsymbol{\Omega}\left(\boldsymbol{\psi}_{i}\right)$.

The amplitude weighted damping is shown in Fig. 10. At the edge of the operational envelope, an increase in cable length causes oscillation with low damping at the PCC, while a short cable and fast WT voltage control dampen the oscillations. A longer cable equals a larger impedance, and the PPC integration constant must follow to increase damping.

\section{Possible Causes for Observed Oscillation}

The results of Section IV showed that flawed tuning of the WT voltage control alone is able to provoke poorly damped local reactive power oscillations, as shown in Fig. 6. Furthermore, an increase in voltage control bandwidth could destabilize the cascade if the current control cannot track its reference. As the system employs rate and output limits, this could lead to limit cycles. An insinuation of this being a contributing mechanism is that the oscillations shown in Fig. 2 were attenuated when WTs were disconnected from the PPC. The PPC tuning bounds of Section V-B show a correlation between WT voltage control performance and PPC sampling time, and aggressive WT voltage control and neglected group transmission delay in the PPC tuning can combined cause the PPC-WT cascade to show oscillatory behavior.

Time domain simulation of the range of stabilizing WT VSI, PPC, and STATCOM control parameters was done in Section VI, and the damping was plotted in Fig. 10. Fig. 10 shows that the electrical distance separating the WTs and STATCOM has a large impact on the damping. In line with Proposition II, the analytical stability bounds show dependence on grid impedance and are subject to uncertainty. 
Settling- and rise-time requirements for the reactive power response at the PCC during a voltage change are specified in the grid code. One method to meet the specification is a large PPC integrator gain. The PPC response in turn is dependent on the impedance and, on WPPs with long array, export, and substation cables, oscillatory behavior can be the result, as we have shown in Section VI. The cable length in the particular is very long for the WPP case referred to in Fig. 2. Fig. 2 shows these observed oscillations compared with a simulation of a WPP with very long cables and a PPC integral gain increased to fulfill the grid code rise time parameter. It is thus plausible that the observed oscillations are caused by the mechanism described and that they can be very poorly damped when having long cables. The grid code specification may, therefore, be difficult to achieve with the applied strategy for voltage control. Furthermore, if the WT and STATCOM voltage control are tuned according to an aggressive grid code [32], the impact of the system impedance variation with control parameter perturbation shows a possible explanation for the observed phenomena.

\section{CONCLUSION}

The reactive power oscillations encountered when energizing the WPP caused quite a bit of concern as they could not be replicated by simulation software. Identification of problem causes in systems with multiple controllers poses many challenges. Without direct access to the implicated systems, a bottom-up approach was necessary. This brief, therefore, tied the analytical properties of WT and STATCOM control design to a generalized filter setup to assess the performance and disturbance rejection properties.

The voltage control parameter stability envelopes of WT and STATCOM were derived as Hurwitz signatures, as the functions of control and system parameters. Based on the results for WT and STATCOM voltage control, stability guidelines for the PPC were proposed to ensure proper cooperation between STATCOM and WT control, considering sampling frequency, droop, and network voltage sensitivity. Analytical assessment of the WPP voltage control was shown to be feasible by considering two joint cascade control systems within the complex control topology and model these as an Multi Input Single Output (MISO) system. The system was simulated over a realistic envelope of electrical impedances and control parameters and it was shown that the oscillatory responses, which were observed, could be reproduced using perturbed parameters in WT voltage control and in the PPC control.

The main result of the investigation was to show that, assuming a stable combination of WT and STATCOM cascade control, oscillations at the PCC could be caused by a too aggressive PPC setting for the system at hand, according to Proposition II. Proposition I was shown to be plausible, but having only a minor contribution to PCC oscillations. Achieving oscillations similar to observed data is not evidence of root cause, but should be seen as proof of possible risks. The methods developed in this brief should be seen as contributions to mitigate such risk and help avoid similar problems in future
WPPs. Finally, this brief contributed by demonstrating how the applied voltage control strategy could be modified to decouple the cascaded control systems.

\section{REFERENCES}

[1] M. G. Simões and S. Chakraborty, Power Electronics for Renewable and Distributed Energy Systems: A Sourcebook of Topologies, Control and Integration, S. Chakraborty, M. G. Simões, and W. E. Kramer, Eds. London, U.K.: Springer-Verlag, 2013.

[2] J.-J. Jung, H.-J. Lee, and S.-K. Sul, "Control strategy for improved dynamic performance of variable-speed drives with modular multilevel converter," IEEE J. Emerg. Sel. Topics Power Electron., vol. 3, no. 2, pp. 371-380, Jun. 2015.

[3] L. Fan, H. Yin, and Z. Miao, "On active/reactive power modulation of DFIG-based wind generation for interarea oscillation damping," IEEE Trans. Energy Convers., vol. 26, no. 2, pp. 513-521, Jun. 2011.

[4] V. Calderaro, G. Conio, V. Galdi, and A. Piccolo, "Reactive power control for improving voltage profiles: A comparison between two decentralized approaches," Electr. Power Syst. Res., vol. 83, no. 1, pp. 247-254, 2012.

[5] D. F. Opila, A. M. Zeynu, and I. A. Hiskens, "Wind farm reactive support and voltage control," in Proc. iREP Symp. Bulk Power Syst. Dyn. Control, 2010, pp. 1-10.

[6] C. Zheng and M. Kezunovic, "Distribution system voltage stability analysis with wind farms integration," in Proc. North Amer. Power Symp. (NAPS), 2010, pp. 1-6.

[7] J. D. Duan, R. Li, and L. An, "Study of voltage stability in gridconnected large wind farms," Mater. Sci. Inf. Technol., vols. 433-440, pp. 1794-1801, Jan. 2012.

[8] L.-J. Cai and I. Erlich, "Power system static voltage stability analysis considering all active and reactive power controls-Singular value approach," in Proc. IEEE Lausanne Power Tech, Jul. 2007, pp. $367-373$.

[9] J. B. Glasdam, "Harmonics in offshore wind power plants employing power electronic devices in the transmission system," Ph.D. dissertation, Dept. Energy Technol. Faculty Eng. Sci., Aalborg Univ., Aalborg, Denmark, 2015.

[10] A. Berizzi, C. Bovo, V. Ilea, M. Merlo, A. Miotti, and F. Zanellini, "Decentralized reactive power control of wind power plants," in Proc. IEEE Int. Energy Conf. Exhibit., Sep. 2012, pp. 674-679.

[11] M. Hunyar and K. Veszpremi, "Reactive power control of wind turbines," in Proc. 16th Int. Power Electron. Motion Control Conf. Expo., 2014, pp. $348-352$.

[12] A. Ghaffari, M. Krstic, and S. Seshagiri, "Power optimization and control in wind energy conversion systems using extremum seeking," IEEE Trans. Control Syst. Technol., vol. 22, no. 5, pp. 1684-1695, Sep. 2014.

[13] P. Guo, W. Y. Liu, W. Wang, N. B. Wang, and H. S. Jia, "Wind farm voltage control scheme to improve dynamic voltage stability," $A d v$. Mater. Res., vols. 732-733, pp. 745-751, Aug. 2013.

[14] T. Friedli, J. W. Kolar, J. Rodriguez, and P. W. Wheeler, "Comparative evaluation of three-phase AC-AC matrix converter and voltage DC-link back-to-back converter systems," IEEE Trans. Ind. Electron., vol. 59, no. 12, pp. 4487-4510, Dec. 2012.

[15] T. Messo, J. Jokipii, and T. Suntio, "Effect of conventional grid-voltage feedforward on the output impedance of a three-phase photovoltaic inverter," in Proc. Int. Power Electron. Conf. (IPEC), Hiroshima, Japan, 2014, pp. 514-521.

[16] J. C. Ramos and R. E. Araujo, "Design considerations on feedforward and Kalman tracking filters in grid-tied-inverters currentcontrol," in Proc. IEEE 23rd Int. Symp. Ind. Electron. (ISIE), Jun. 2014, pp. 2099-2104.

[17] A. Dòria-Cerezo, M. Bodson, C. Batlle, and R. Ortega, "Study of the stability of a direct stator current controller for a doubly fed induction machine using the complex Hurwitz test," IEEE Trans. Control Syst. Technol., vol. 21, no. 6, pp. 2323-2331, Nov. 2013.

[18] L. H. Keel and S. P. Bhattacharyya, "Controller synthesis free of analytical models: Three term controllers," IEEE Trans. Autom. Control, vol. 53, no. 6, pp. 1353-1369, Jul. 2008.

[19] R. Vilanova and A. Visioli, Eds., PID Control in the Third Millennium: Lessons Learned and New Approaches. London, U.K.: Springer-Verlag, 2012.

[20] J. Forde and P. Nelson, "Applications of Sturm sequences to bifurcation analysis of delay differential equation models," J. Math. Anal. Appl., vol. 300, no. 2, pp. 273-284, 2004. 
[21] M. Liserre, F. Blaabjerg, and S. Hansen, "Design and control of an LCL-filter-based three-phase active rectifier," IEEE Trans. Ind. Appl., vol. 41, no. 5, pp. 1281-1291, Sep./Oct. 2005.

[22] C. Wessels, J. Dannehl, and F. W. Fuchs, "Active damping of LCL-filter resonance based on virtual resistor for PWM rectifiersStability analysis with different filter parameters," in Proc. IEEE Annu. Power Electron. Specialists Conf., Jun. 2008, pp. 3532-3538.

[23] S. Skogestad and I. Postlethwaite, Multivariable Feedback Control: Analysis and Design, 2nd ed. New York, NY, USA: Wiley, 2009.

[24] Ł. H. Kocewiak, "Harmonics in large offshore wind farms," Ph.D. dissertation, Dept. Energy Technol., Aalborg Univ., Aalborg, Denmark, 2012.

[25] M. Monfared, S. Golestan, and J. M. Guerrero, "Analysis, design, and experimental verification of a synchronous reference frame voltage control for single-phase inverters," IEEE Trans. Ind. Electron., vol. 61, no. 1, pp. 258-269, Jan. 2014.

[26] M. P. S. Gryning, "Offshore wind park control assessment methodologies to assure robustness," Ph.D. dissertation, Tech. Univ. Denmark, Lyngby, Denmark, 2015.
[27] E. Wu and P. W. Lehn, "Digital current control of a voltage source converter with active damping of LCL resonance," IEEE Trans. Power Electron., vol. 21, no. 5, pp. 1364-1373, Sep. 2005.

[28] J. M. Amada and M. P. C. Moreno, "Reactive power injection strategies for wind energy regarding its statistical nature," in Proc. 6th Int. Workshop Large-Scale Integr. Wind Power Transmiss. Netw. Offshore Wind Farms, vol. 138. 2006.

[29] P. Kundur, Power System Stability and Control, New York, NY, USA: McGraw-Hill, 1993.

[30] P. Zhang, R. Li, J. Shi, and X. He, "An improved reactive power control strategy for inverters in microgrids," in Proc. IEEE Int. Symp. Ind. Electron., May 2013, pp. 1-6.

[31] A. Wolfenden and V. K. Kinra, Eds., $M^{3} D$ III: Mechanics and Mechanisms of Material Damping (ASTM Special Technical Publication// Stp). Philadelphia, PA, USA: ASTM, 1997.

[32] C. Sourkounis and P. Tourou, "Grid code requirements for wind power integration in Europe," in Proc. Conf. Papers Energy, 2013, Art. no. 437674. 